\title{
Features and outcomes of secondary sepsis and urinary tract infections in COVID-19 patients treated with stem cell nebulization
}

Authors: All authors participated on the critical revision of the manuscript for important intellectual content

Gina M. Torres Zambrano. Conception and design, acquisition of data. Consultant Urologist at Abu Dhabi Stem Cell Center (ADSCC). Telephone number: +971 506439223. dr.torresz@gmail.com; gina.zambrano@adscc.ae.

Rene Antonio Rivero. Head of ADSCC Scientific Committee. rene.rivero@adscc.ae

Carlos A. Villegas Valverde. Analysis and interpretation of data, statistical analysis. Researcher at the ADSCC Immunology Laboratory. carlos.valverde@adscc.ae

Yendry Ventura Carmenate. ADSCC General Manager. yendry.ventura@adscc.ae

Mailing address: Street \#31, Area Muroor Road, Building \# C43, Al Murjan Tower, Apartment 107, Abu Dhabi, UAE. Abu Dhabi Stem Cells Center (ADSCC) Ringgold ID: 580443.

Keywords: COVID-19; hemodialysis; sepsis; stem cell therapies.

Word count: Of the text: 2697 . Of the abstract: 248 .

\section{Abstract:}

Background: COVID-19 is the defining global crisis of our time. Secondary complication such as urinary tract infections and sepsis, worsen the already established problem, creating a new challenge. Objective: To characterize the features and outcomes in COVID-19 patients with sepsis and urinary tract infection. Methods: An observational and analytical study was conducted within the framework of the SENTAD COVID clinical trial at the Abu Dhabi Stem Cells Center, were the patients received a nebulization therapy with the use of autologous stem cells (group A). Those patients were compared with a not stem cells treated control arm (group B), and both received the UAE COVID 19 standard 
management. An analysis of the culture samples, antimicrobial agents and the efficacy of the therapy on patient's outcomes was done. Results: A significant difference between the groups was found in the UTI incidence $(p=* 0.0206)$. Patients in group A showed a lower tendency to sepsis in comparison with group B ( $7 \%$ vs $21 \%), H R=0.35$, (95\% Confidence Interval: $0.13-0.91), p=0.0175$. It was calculated a NNT=7.3. Candida albicans was the most frequently agent causing sepsis and UTI. The massive use of broadspectrum antimicrobials was striking. Conclusions: We found a protective factor of stem cells against secondary infection in COVID 19 cases, in terms of sepsis and UTI. The suggested immunomodulatory effect of stem cells offers a therapeutic strategy to manage the disease and avoid several complications. Antimicrobial agents can lead to increased opportunistic infections, so a rational approach to these treatments must be considered.

\section{Acknowledgments:}

Acknowledgment to the government of the United Arab Emirates and especially to the Abu Dhabi Health Services Company SEHA for the contribution and support to this study.

\section{Introduction:}

The corona virus disease (COVID-19) is the defining global crisis of our time and the greatest threat this world has faced in a long time. Many countries, including the UAE are taking every precautionary measure to slow and control the spread. ${ }^{1}$

Taking into account that the sepsis and septic shock contribute to the cytokine storm syndrome causing a high mortality rate $(60-90 \%)^{2}$ and also generate an immune-mediated kidney damage, ${ }^{2,3}$ it is crucial to prevent this event. Studies showed that there was a sharp increase in Methicillin-resistant Staphylococcus aureus (MRSA), Pseudomonas and Candida species infectious rate among the intensive care unit (ICU) COVID-19 patients, indicating that secondary infection affect the prognosis and subsequent treatment of these patients. ${ }^{4}$

Given the immunomodulatory potential of the stem cells in sepsis and clinical evolution of chronic conditions with the stimulation by humoral factors, strategies such as stem cell-based therapy are being proposed to regulate inflammation, prevent or mitigate this cytokine storm through their immunomodulatory capacity. ${ }^{5}$

The Abu Dhabi Stem Cell Centre (ADSCC) research team developed a treatment for COVID-19 ("SENTAD COVID Study" clinical trial) with the use of an autologous stem cells cocktail, called Peripheral Blood Non-Hematopoietic Enriched Stem Cells (PBNHESC), that has shown promising results. ${ }^{6}$ Previously, we studied the frequency of acute renal injury (AKI) in patients with COVID19 and their relation with clinical outcomes within the framework this clinical trial, finding it in about a third of critically ill patients. In addition, those who received the treatment showed a better tendency to improve in terms of hospital stay and biomarker evolution (amelioration of 
linphopenia, ${ }^{7-9}$ neutrophil-lymphocyte ratio ${ }^{10-13}$ and C-reactive protein (CRP)) than those of the control group.

Scientist and researchers all over the world are racing to find treatment because not only is COVID-19 a major health crisis, but it also has the potential to create devastating social, economic and political catastrophes to every country it touches. In this article we analysed the incidence of sepsis and urinary tract infection (UTI) and related pathogens, in comparison with the rest of the germs that cause sepsis in the two groups of patients of our study, after receiving the treatment or being recruited as a control.

\section{Objective:}

To characterize the features and outcomes in COVID-19 patients with sepsis and urinary tract infection after intervention, within the framework of the ADSCC SENTAD COVID clinical trial at the Abu Dhabi Emirate, during the month of April of 2020.

\section{Methods:}

Within the framework of the SENTAD COVID Study clinical trial, ${ }^{6}$ we performed a multi-center, prospective and analytical study to figure out the sepsis and UTI incidence among the patients after the intervention. Four different hospitals from the Emirate of Abu Dhabi participated: Sheikh Khalifa Medical City, Al Rahba Hospital, Al Mafraq Hospital and Al Ain Hospital.

From the main study, a total of 139 patients were divided in two groups for the comparison:

1. Group A (Experimental arm) COVID 19 standard care plus nebulization with PBNHESC $(n=69)$

2. Group B (No intervention arm) COVID 19 standard care $(n=70)$.

Both groups fulfil the inclusion criteria: Aged $\geq 18$ years, laboratory confirmation of COVID-19, interstitial lung change judged by computed tomography, hospitalized and symptomatic patients, referring one or more symptoms, ability to comply with test requirements and blood collection and agrees to participate in the study.

The exclusion criteria were: Patients with diagnosis of any kind of shock, organ transplants in the past 3 months, patients receiving immunosuppressive therapy, diagnostic of Hepatitis B Virus (HBV) infection or Human Immunodeficiency Virus (HIV) infection or Acquired Immunodeficiency Syndrome (AIDS), current diagnosis of cancer or history of malignancies in the past 5 years, pregnant or lactating women, patients who had participated in other clinical trials in the past 3 months, inability to comply with test requirements and blood collection or inability to provide informed consent. 
In order to determine the clinical severity, the ordinal scale created by the WHO committee for COVID -19, that measures the illness severity overtime, was used: ${ }^{14}$ (Table 1 )

Table 1. SENTADO-COVID Study seven-category ordinal scale for clinical involvement.
\begin{tabular}{|l|l|}
\hline Category & Score \\
\hline No limitation of activities, discharged from hospital & 1 \\
\hline Limitation of activities. & 2 \\
\hline Hospitalized, no oxygen therapy. & 3 \\
\hline Oxygen by mask or nasal prongs. & 4 \\
\hline Non-invasive ventilation or high-flow oxygen & 5 \\
\hline Intubation and mechanical ventilation. & 6 \\
\hline Mechanical Ventilation + additional organ support: ECMO, CRRT, vasopressors. & 7 \\
\hline Death & 8 \\
\hline
\end{tabular}

A registration of gender, age, BMI, and comorbidities, vital signs, and biochemical studies performed within $24 \mathrm{~h}$ of inclusion in the trial was done. Both groups received COVID 19 standard treatment defined as "UAE National Guidelines for Clinical Management and Treatment of COVID 19, v.2.0" as per the Department of Health (DOH) ${ }^{1}$. A stem cell nebulization with autologous PBNHESC-C (cocktail rich in very small embryonic-like stem cells (VSELs) and platelets rich plasma derived growth factors, commercially named UAECell 19), was given by two nebulization of $10 \mathrm{cc}$, in two consecutive days (at least 22 hours between each one) for patients at group A, obtained through phlebotomy of $300 \mathrm{cc}$ and processed at the ADSCC laboratory, and cells were characterized by flow cytometry and inverted automated fluorescent microscopy.

Follow up of both groups were done until the discharge. Secondary infection was diagnosed when the patients had clinical symptoms with a positive culture (blood, sputum, others) and UTI with a positive urine culture. Consequently, the reports of the sputum, blood, specific seeking of Methicillin-resistant Staphylococcus aureus (MRSA), skin/wound and urine cultures were collected, as well as the antimicrobial treatment given.

Statistical analysis: a non-normal distribution of the variables was found, so non-parametric statistical methods were used. A proportions comparison test (Chi-Square) for the body mass index (BMI) categories ${ }^{15}$ and comorbidities, and U-Mann-Whitney for the dates intervals. The Hazard Ratio (HR), 95\% Confidence Interval, Number Needed to Treat (NNT) was calculated for the sepsis complication. A significance level of $p<0.05$ was prefixed.

Ethics: The study was approved by the Emirates Institutional Review Board for COVID-19 Research (Ref. ID: DOH/CVDC/2020/1172). Study participants provided written informed consent per the Helsinki Declaration. ${ }^{16}$ The consent document described the importance of participation and explained the study's characteristics and possible risks and benefits. All data were kept confidential and participant identity was delinked. The selection of diagnostic tools 
followed the ethical principles of maximum benefit and non-maleficence. This work was supported by ADSCC. This research had not received any other specific grant from any funding agency in the public, commercial or not-for-profit sectors. Award/Grant number is not applicable. None of the contributing authors have any conflicts of interest. For extra data: dr.torresz@gmail.com.

\section{Results:}

From the 139 patients, there were 129 males (93\%) and 10 females (7\%), 65 males (94\%) from the group A and 64 (91\%) from the group B, and 4 females (6\%) vs. $6(9 \%)$ correspondingly. The ages of group A ranged from 27 to 71 years old (mean 45.9 years old), and from 26 to 73 years old in group B (mean 44.31 years old), with no significant differences between the two groups $(p=0.3677)$. The distribution of the BMI was between 17.06 to 45.44 (mean 27.61) in the group in the group $A$, and between 16.95 to $47.75 \mathrm{~B}$ (mean 27.93), although there were more patients with overweight in group $A$. (Table 2)

Diabetes Mellitus and hypertension were the most commons comorbidities in both groups. No differences were found between the groups regarding those variables. There were more smokers in the group A ( $1 \%$ vs $\left.9 \%,{ }^{*} p=0.0306\right)$. Likewise, there were no differences in the scores between the groups. Table 2 is a summary of the data.

Table 2. Clinics characteristics of the patients.

\begin{tabular}{|c|c|c|c|c|c|}
\hline & \multicolumn{2}{|c|}{ Group A } & \multicolumn{2}{|c|}{ Group B } & \multirow[t]{2}{*}{$\mathbf{p}^{*}$} \\
\hline & $\mathbf{n}$ & $\%$ & $\mathbf{n}$ & & \\
\hline \multicolumn{6}{|l|}{ BMI: } \\
\hline Overweight (25-29) & 35 & $51 \%$ & 19 & $27 \%$ & 0.0038 \\
\hline Mild obesity (30-34) & 11 & $16 \%$ & 13 & $19 \%$ & 0.6429 \\
\hline Moderate obesity (35-40) & 0 & $0 \%$ & 3 & $4 \%$ & 0.0945 \\
\hline Morbid obesity $(>40)$ & 3 & $4 \%$ & 4 & $6 \%$ & 0.5901 \\
\hline \multicolumn{6}{|l|}{ Comorbidities } \\
\hline Smoker & 6 & $9 \%$ & 1 & $1 \%$ & 0.0306 \\
\hline DM & 18 & $26 \%$ & 13 & $19 \%$ & 0.3246 \\
\hline HTN & 18 & $26 \%$ & 19 & $28 \%$ & 0.7913 \\
\hline $\mathrm{DL}$ & 5 & $7 \%$ & 7 & $10 \%$ & 0.5277 \\
\hline Cardiac diseases & 1 & $1 \%$ & 2 & $3 \%$ & 0.4023 \\
\hline Respiratory diseases & 4 & $6 \%$ & 3 & $4 \%$ & 0.5896 \\
\hline \multicolumn{6}{|l|}{ Score } \\
\hline 3 & 36 & $52 \%$ & 40 & $59 \%$ & 0.4080 \\
\hline 4 & 13 & $19 \%$ & 6 & $7 \%$ & 0.0252 \\
\hline 5 & 3 & $4 \%$ & 6 & $10 \%$ & 0.0960 \\
\hline
\end{tabular}


The median hospital stay after receiving the treatment in the study for group A was 5 days, and 6 days in group B after being included, but the range for group B was from 2 to 125 days, in compared with 1 to 43 days in group $A(p=0.2924)$. Apropos the UTI incidence, a significant difference between the groups was found $(p=0.0206)$. Patients in group A showed a lower tendency to sepsis in comparison with group B (7\% vs $21 \%$ ), HR=0.35, (95\% Confidence Interval: $0.13-0.91), p=0.0175$. It was calculated a NNT=7.3. In group $B$ there were 6 deaths while in the group A there were only 4. (Table 3 )

Table 3. Clinical outcome characterizations.

\begin{tabular}{|l|c|c|c|c|c|}
\hline \multirow{2}{*}{\multicolumn{1}{|c|}{ Event }} & \multicolumn{2}{|c|}{ Group A } & \multicolumn{2}{c|}{ Group B } & \multirow{2}{*}{ p } \\
\cline { 2 - 6 } & $\mathbf{n}$ & $\mathbf{\%}$ & $\mathbf{n}$ & $\%$ & $\mathbf{0 . 0 2 0 6}$ \\
\hline UTI & 1 & $1 \%$ & 7 & $10 \%$ & $\mathbf{0 . 0 1 7 5}$ \\
\hline Sepsis & 5 & $7 \%$ & 15 & $21 \%$ & 0.6434 \\
\hline Mortality & 4 & $6 \%$ & 6 & $8 \%$ & \\
\hline
\end{tabular}

*: proportions comparison test. UTI: urinary tract infection.

Cultures were obtained from all the critically ill patients, 23 from group B and 20 from group A, 41 samples from blood ( 23 vs. 18), 23 from urine ( 22 vs. 1), 31 MRSA ( 22 vs. 9), 29 from sputum (15 vs. 14) and 2 from wound/skin ( 2 vs. 0). In total 39 patient's samples (28\%) identified at least one pathogen. From group B, 27 (38\%) samples were positive: 9 from blood (39\%), 8 from urine (36\%), 6 from sputum (40\%), 1 MRSA (5\%) and 3 from wound/skin (100\%). From group A, 12 samples were positive (17\%), 5 from blood (28\%), 1 from urine (5\%), 6 from sputum (43\%).

Candida albicans caused 21\% of the sepsis in both groups (7 from group B vs 2 from group A), Staphylococcus aureus 19\% (5 vs 3 cases), Pseudomonas aeruginosa $16 \%$ (3 vs 4 cases), Streptococcus pneumonia 5\% (1 vs 1 case), Klebsiella pneumoniae 9\% (4 vs 0 cases), Escherichia coli $9 \%$ ( 4 vs 0 cases) and Enterobacter aerogenes 2\% (1 vs 0 cases). From the group A, 6 patients with UTI were found to have other positive cultures, 3 from blood, 2 from sputum and 1 had MRSA. The patient from group A who had UTI had sepsis caused by Candida albicans. Of the deceased patients, only one from group B had a UTI (14\%) caused by Klebsiella pneumoniae, none from group A. (Figure 1)

In 11 cases more than one pathogen grew, 9 from group B and 2 from group A, where Candida albicans was the most frequently found concomitant pathogen along with other germs (5 vs.2), 
followed by Staphylococcus aureus (3 vs. 1), Klebsiella pneumonia (3 vs. 0) and Pseudomonas aeruginosa (2 vs. 1). (Table 4)

Table 4. Multiple pathogens infection distribution.

\begin{tabular}{|lcc|}
\hline \multicolumn{1}{|c}{ Pathogens } & Group & Group \\
Candida albicans + Pseudomonas aeruginosa & A & B \\
Candida albicans + Staphylococcus aureus & 1 & 1 \\
Candida albicans + Klebsiella pneumoniae & 1 & 1 \\
Candida albicans + Streptococcus pneumoniae & & 1 \\
Candida albicans + Enterobacter aerogenes & 1 \\
Klebsiella pneumoniae + Acinetobacter baumannii & 1 \\
Streptococcus pneumoniae + Klebsiella pneumoniae + Escherichia coli & 1 \\
Staphylococcus aureus + Escherichia coli & 1 \\
Pseudomonas aeruginosa + Staphylococcus aureus + Enterobacter aerogenes & \\
Total & 2 & 1 \\
\hline
\end{tabular}

Regarding the use of antibiotics, 60 pacients received this therapy, 30 from each group. Piperacillin/tazobactam was the most commonly used in both groups, followed by Meropenem, Vancomicin and Linezolid. (Figure 2). It was marked the use of several antimicrobial agens in 42 patients (30\%), 17 from group A (25\%) and 25 patients from group B (35\%), from those, 11 received 2 combination of antibiotics (8\%, 9 from group A and 7 from group B ), 15 received 3 $(11 \%, 11$ vs. 4), 6 received 4 (4\%, 4 vs. 2$), 3$ received 5 (2\%, 3 vs. 0$)$ and 2 received $6(1 \%, 2$ vs. 0).

On the subject of the antifungical treatment, 9 patient received treatment, 2 from group $A$ and 7 from group B. From the second group, 1 patient received 2 antifungical (this patient received also treatment with linezolid and meropenem). The most frequently used in group $B$ was anidalafungin and caspofungin was in group A. (Figure 2)

\section{Discussion:}

The data analyzed from this study showed a higher incidence of COVID-19 among men, especially among critically ill patients (no women with high scores were found during the study period). Other authors confirmed male predominance in the incidence of this disease compared to female, it has also been found that patients with diabetes, hypertension, coronary heart 
disease, chronic obstructive pulmonary disease, cerebrovascular disease, and kidney disease exhibit worse clinical outcomes when are infected with COVID-19 ${ }^{17,18}$. Similarly, the patients included in the study were found to have this comorbidities as risk factors forsepsis. ${ }^{19}$ Despite the fact that among the treated patients there were more smokers and more overweight cases, it was found that infectious complications were lower than those of the control group.

Bacterial and fungal secondary infection rate in hospitalized adults with COVID-19 is poorly understood, and are associated with intensive care unit admission and higher risk of invasive procedures ${ }^{4}$. As a result, secondary infection might be a key element that leads to severe disease and mortality. In our study, there was high culture sampling rate among he critically ill patients in both arms, with an important role of the Candida albicans causing sepsis (21\% of cultures) and was the most common germ in association with other bacterial infections, but treated patients were less prone to this event than controls, with a statically significant difference, and it was found that around 7 patients are required to be treated to have this protective effect with the stem cell nebulization (NNT=7.3). Surprisingly, despite the fact that all septic patients had a urinary catheter, the rate of UTI was not strikingly high in group A compared with group B. An attenuation of the bacterial sepsis stem cell mediated had been described, via several mechanisms such as improving the phagocytic ability, secreting antimicrobial peptides ${ }^{5,20}$, and increasing bacterial clearance. ${ }^{5,21}$

The COVID-19 sepsis can be associated with an immunosuppresive effect of the SARS-Cov- 2 and for instance, as shownin this study C Albicans, as well as Pseudomonas, are more frequenty found as oportunistic patogen associted with a depressed immune response. Taking into count that a large proportion of patients had received therapy with one or more broadspectrum antibacterials at the time of culture, in some cases up to 6 antibiotics, the reason of the fungal sepsis could be related to these managements. Other authors also found evidenced a strong increase in opportunistic infections rate among SARS ICU patients, due to MRSA, Pseudomonas, and Candida species. ${ }^{4,22}$ There is being described the biofilm formation as a resistant mediator, especially in those with MRSA positive culture, which is a survival mechanism, since it constitutes a reservoir of bacteria with multi-resistance. ${ }^{23,24}$ The antibacterial therapy, if indicated, should be prescribed in line with local guidelines and reviewed with clinical response at 48 to 72 hours. If no evidence of bacterial coinfection is found, then stopping antibacterial therapy should be considered. ${ }^{25}$ It is well known that the prolonged use of antimicrobials and their inappropriate use, contribute to the development of major consequences, and we are facing a lack of data about antimicrobial use during this pandemic worldwide in just a few months, then the emergence of new resistance to this treatmets should be expected, without counting other adverse effects of these therapies for kidney and liver function for example, with unimaginable implications for human and animal health and the environment. ${ }^{26}$

Another reason of these secondary infections must be related with the lymphopenic status of the critically ill COVID-19 patients already found in the main study ${ }^{6}$. There is a sustained and substantial reduction of the peripheral lymphocyte counts, especially CD4 T and CD8 T cells, as 
the representative of immune suppression stage after the cytokine storm activation phase increasing the risk of developing secondary infection ${ }^{4,27}$.

Even though the median hospital stay after the intervention was not significant between the groups, the range is much shorter in group A patients than in group B, given the homogeneity of the treated group due to the therapy, and the statistical dispersion of the data of the control group, giving another hypothesis of the lower incidence of sepsis and UTI in group A, since the shorter hospital stay is a protective factor against nosocomial infections ${ }^{28}$.

Moreover, due to the autologous origin of the therapy, there are no immunologic reactions, with rare adverse effects more linked with the collection of the blood (fainting and dizziness may occur).

There are some limitations to the current study. It was carried in 4 different hospitals, each one with different criteria for taking cultures, as well the selection of the initial empiric antimicrobials treatment, can cause multiple germ resistant and negativization of some of the samples. It is our responsability for implementing and developing actions to control or stop the disease, but also change dangerous practices, through appropriate and consensual use of antimicrobials.

\section{Conclusion:}

In this study, we found a protective factor of stem cells against secondary infection in COVID 19 cases, in terms of sepsis and UTI. Patients in the treated group showed a lower tendency to develop these events compared to the control group. The suggested immunomodulatory effect of stem cells offers a therapeutic strategy to manage the disease and avoid several complications, becoming a crucial adjuvant tool for healing and achieving early recovery in severe COVID-19 infections. It is well known that during this disease, patients had received several antimicrobial agents that can lead to increased fungal and opportunistic infections, so a rational approach to these treatments should be considered.

\section{Take Home Message:}

There is a significant frequency of sepsis and UTI as complications in COVID-19, related to the antimicrobial use, length of hospital stays and invasive procedures. Novel stem cell nebulization therapy may improve the clinical evolution and frequency of this complications.

\section{Bibliography}

1. National Clinical Committee for COVID-19 Management. National Guidelines for Clinical Management and Treatment of COVID-19 1 st June, 2020 Version 4.0. United Arab Emirates Minist Heal. 2020;19(V4):1-61. 
https://www.dha.gov.ae/en/HealthRegulation/Documents/National_Guidelines_of_COVID_19_1 st_June_2020.pdf

2. Naicker S, Yang CW, Hwang SJ, Liu BC, Chen JH, Jha V. The Novel Coronavirus 2019 epidemic and kidneys. Kidney Int. 2020;97(5):824-828. doi:10.1016/j.kint.2020.03.001

3. Puliatti S, Eissa A, Eissa R, et al. COVID-19 and urology: a comprehensive review of the literature. BJU Int. 2020;125(6):E7-E14. doi:10.1111/bju.15071

4. Zhang $\mathrm{H}$, Zhang $\mathrm{Y}, \mathrm{Wu}$ J, et al. Risks and features of secondary infections in severe and critical ill COVID-19 patients. Emerg Microbes Infect. 2020;9(1):1958-1964.

doi:10.1080/22221751.2020.1812437

5. Majolo F, da Silva GL, Vieira L, Timmers LFSM, Laufer S, Goettert MI. Review of Trials Currently Testing Stem Cells for Treatment of Respiratory Diseases: Facts Known to Date and Possible Applications to COVID-19. Stem Cell Rev Reports. Published online 2020. doi:10.1007/s12015020-10033-6

6. Study Evaluating the Safety and Efficacy of Autologous Non-Hematopoietic Peripheral Blood Stem Cells in COVID-19 - Full Text View - ClinicalTrials.gov. Accessed October 18, 2020. https://clinicaltrials.gov/ct2/show/study/NCT04473170?term=stem+cell\&cond=covid\&cntry=AE \&draw $=2 \&$ rank=1

7. Frater JL, Zini G, d'Onofrio G, Rogers HJ. COVID-19 and the clinical hematology laboratory. Int J Lab Hematol. 2020;42(S1):11-18. doi:10.1111/ijlh.13229

8. Lippi G, Plebani M. Laboratory abnormalities in patients with COVID-2019 infection. Clin Chem Lab Med. 2020;58(7):1131-1134. doi:10.1515/cclm-2020-0198

9. Fan BE, Chong VCL, Chan SSW, et al. Hematologic parameters in patients with COVID-19 infection. Am J Hematol. 2020;95(6):E131-E134. doi:10.1002/ajh.25774

10. Akilli NB, Yortanli M, Mutlu H, et al. Prognostic importance of neutrophil-lymphocyte ratio in critically ill patients: Short- and long-term outcomes. Am J Emerg Med. 2014;32(12):1476-1480. doi:10.1016/j.ajem.2014.09.001

11. Basbus L, Lapidus MI, Martingano I, Puga MC, Pollán J. Neutrophil to lymphocyte ratio as a prognostic marker in COVID-19. 2020;80:31-36.

12. Witjes JA, Bruins M, Cathomas R, et al. EAU Guidelines on Muscle-invasive and metastatic Bladder Cancer 2020. Eur Assoc Urol Guidel 2020 Ed. 2020;presented. http://uroweb.org/guideline/bladder-cancer-muscle-invasive-and-metastatic/ LK - Muscleinvasive and Metastatic Bladder Cancer Uroweb\%7Chttp://uroweb.org/guideline/bladdercancer-muscle-invasive-and-metastatic/\%7C FG - 0

13. Ljungberg B, Albiges L, Bensalah K, et al. EAU Guidelines. Edn. presented at the EAU Annual Congress Amsterdam 2020. Eur Urol. 2020;67(5):913-924.

14. WHO R\&D Blueprint Novel Coronavirus COVID-19 Therapeutic Trial Synopsis.; 2020.

15. U.S. Department of Health and Human Services. Managing Overweight and Obesity in Adults: Systematic Evidence Review from the Obesity Expert Panel. Natl Hear Lung, Blood Inst. Published online 2013:501. https://www.nhlbi.nih.gov/sites/default/files/media/docs/obesity-evidence- 
review.pdf

16. World Medical Association declaration of Helsinki: Ethical principles for medical research involving human subjects. JAMA - J Am Med Assoc. 2013;310(20):2191-2194. doi:10.1001/jama.2013.281053

17. Martinez-Rojas MA, Vega-Vega O, Bobadilla XNA. Is the kidney a target of SARS-CoV-2? Am J Physiol - Ren Physiol. 2020;318(6):F1454-F1462. doi:10.1152/AJPRENAL.00160.2020

18. Sonja A. Rasmussen, MD, MS JCS. Treatment for severe acute respiratory distress syndrome from COVID-19. Ann Oncol. 2020;(March 20):518. doi:https://doi.org/10.1016/ S2213-2600(20)301272

19. Eguia E, Cobb AN, Baker MS, et al. Risk factors for infection and evaluation of Sepsis-3 in patients with trauma. Am J Surg. 2019;218(5):851-857. doi:10.1016/j.amjsurg.2019.03.005

20. Krasnodembskaya A, Song Y, Fang X, et al. Antibacterial effect of human mesenchymal stem cells is mediated in part from secretion of the antimicrobial peptide LL-37. Stem Cells.

2010;28(12):2229-2238. doi:10.1002/stem.544

21. Mei SHJ, Haitsma JJ, Dos Santos CC, et al. Mesenchymal stem cells reduce inflammation while enhancing bacterial clearance and improving survival in sepsis. Am J Respir Crit Care Med. 2010;182(8):1047-1057. doi:10.1164/rccm.201001-00100C

22. Yap FHY, Gomersall CD, Fung KSC, et al. Increase in methicillin-resistant Staphylococcus aureus acquisition rate and change in pathogen pattern associated with an outbreak of severe acute respiratory syndrome. Clin Infect Dis. 2004;39(4):511-516. doi:10.1086/422641

23. Fernández-Abreu, Anabel, Bravo-Fariñas, Laura del Carmen, Rivero-Navea, Giselle, NuñezFernández, Fidel Angel, Cruz-Infante, Yanaika, Águila-Sánchez, Adalberto, \& Hernández-Martínez $\mathrm{JL}$. Determination of biofilms and extended-spectrum beta-lactamases in Vibrio cholerae non-01, non-0139 isolates from patients with diarrhea in Cuba. Rev Cubana Med Trop. Published 2019. Accessed November 24, 2020. http://scielo.sld.cu/scielo.php?script=sci_arttext\&pid=S0375$07602019000200011 \&$ lng=es\&nrm=iso

24. Nadell CD, Drescher K, Wingreen NS, Bassler BL. Extracellular matrix structure governs invasion resistance in bacterial biofilms. ISME J. 2015;9(8):1700-1709. doi:10.1038/ismej.2014.246

25. Townsend L, Hughes $G$, Kerr $C$, et al. Bacterial pneumonia coinfection and antimicrobial therapy duration in SARS-CoV-2 (COVID-19) infection. JAC-Antimicrobial Resist. 2020;2(3):1-4. doi:10.1093/jacamr/dlaa071

26. Miranda C, Silva V, Capita R, Alonso-Calleja C, Igrejas G, Poeta P. Implications of antibiotics use during the COVID-19 pandemic: present and future. J Antimicrob Chemother. 2020;75(12). doi:10.1093/jac/dkaa350

27. Zou L, Ruan F, Huang M, et al. SARS-CoV-2 Viral Load in Upper Respiratory Specimens of Infected Patients. N Engl J Med. Published online 2020. doi:10.1056/NEJMc2001737

28. Jeon CY, Neidell M, Jia H, Sinisi M, Larson E. On the Role of Length of Stay in HealthcareAssociated Bloodstream Infection. Infect Control Hosp Epidemiol. 2012;33(12):1213-1218. doi:10.1086/668422 
medRxiv preprint doi: https://doi.org/10.1101/2020.12.05.20244483; this version posted December 7, 2020. The copyright holder for this preprint (which was not certified by peer review) is the author/funder, who has granted medRxiv a license to display the preprint in perpetuity.

\author{
It is made available under a CC-BY-NC-ND 4.0 International license.
}


A)
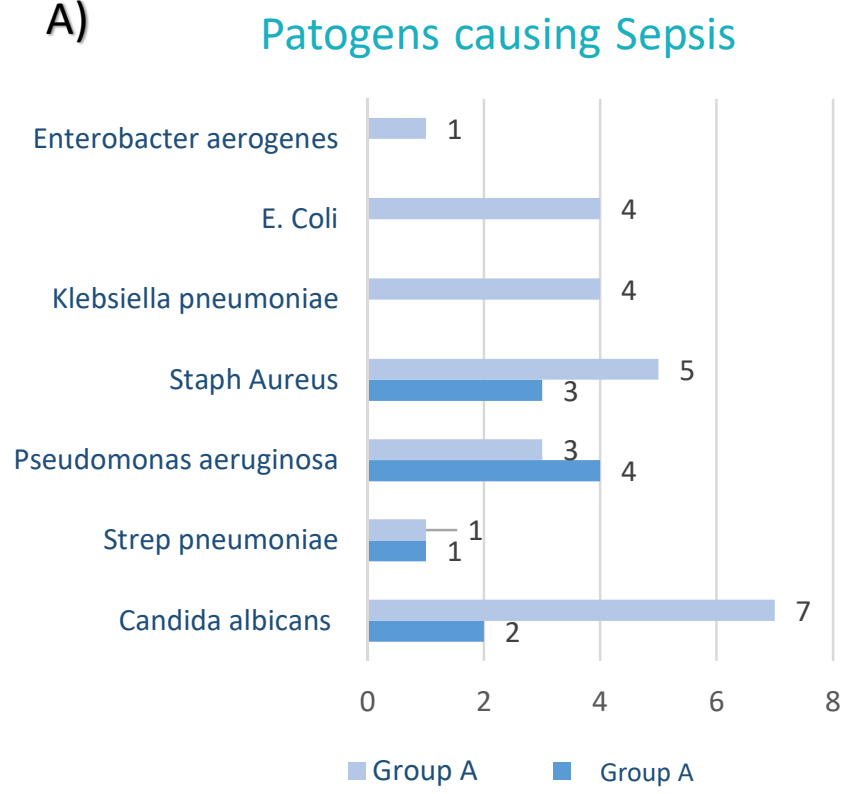

\section{B) Urine culture}

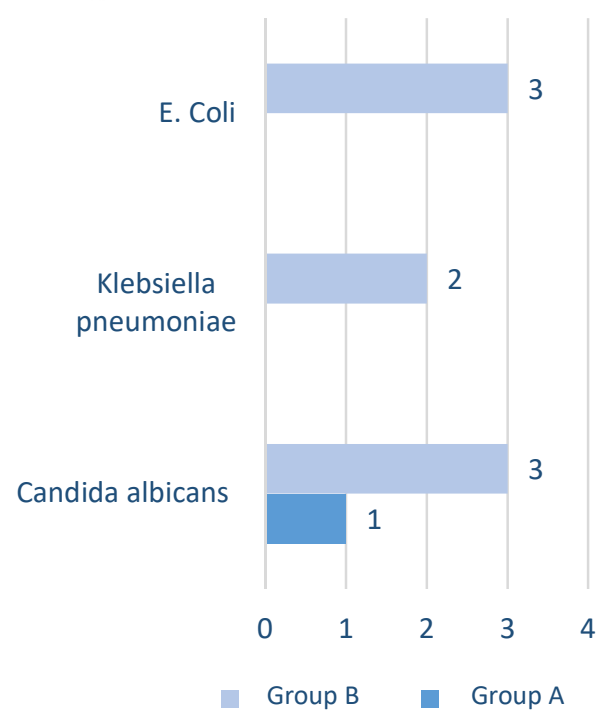

Figure 1. Cultures reports: A) Number of cases with pathogens found in different cultures $Y$ B) Number of cases with pathogens grown in urine cultures. 


\section{A) Antimicrobial Treatment}

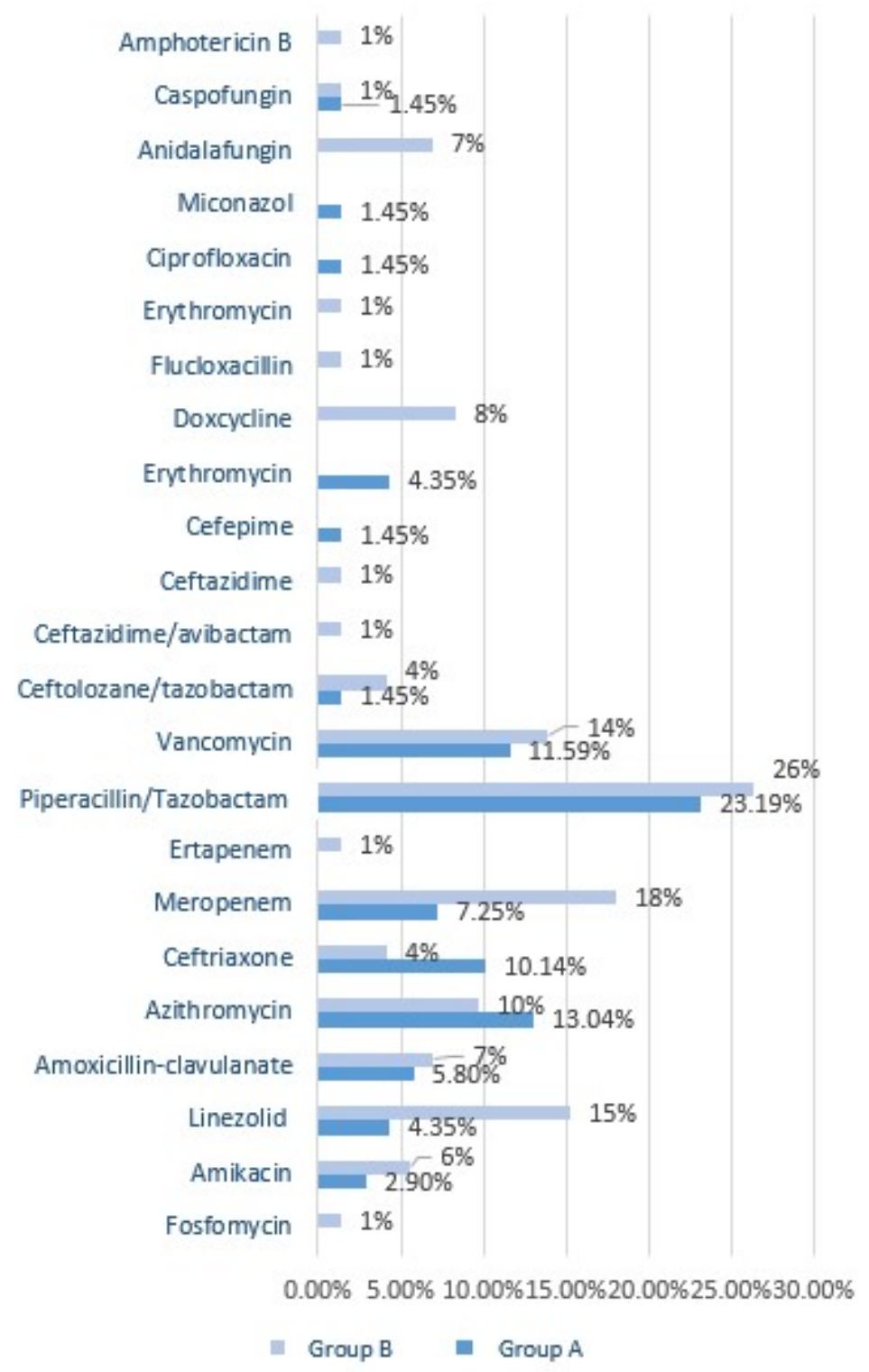

B) Antimicrobial for

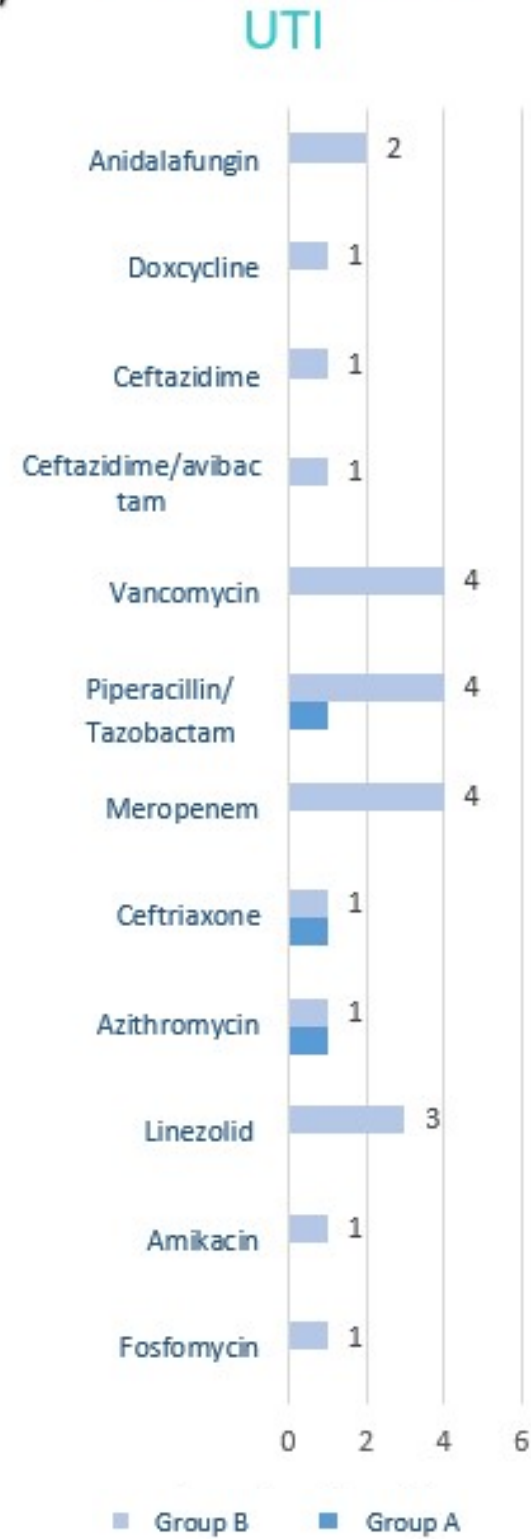

Figure 1. Antimicrobial treatment. A) Treatment for from both groups. B) Treatment for patients with UTI. 